\title{
Manikin-Based Simulation: an Update to the Clerkship Experience
}

\author{
Sarah E. Baker ${ }^{1}$ (D) Kristin Escamilla $^{2} \cdot$ Mallory Jacobs $^{1} \cdot$ Rachel Sherhart $^{3} \cdot$ Kathlene Trello-Rishel $^{1} \cdot$ Brian Fuehrlein $^{4}$
}

Received: 6 April 2021 / Accepted: 28 April 2021 / Published online: 18 May 2021

(C) Academic Psychiatry 2021

To the Editor:

High-fidelity manikin-based simulation (MBS) was introduced into the psychiatry clerkship experience at Yale University several years ago [1]. Despite its growing popularity and use in other specialties, this technology is not widely incorporated into psychiatry education [2]. Our group wished to study student attitudes toward this technologically driven educational tool as part of a psychiatry clerkship experience to understand if it would be well received by students and could be utilized more widely.

As part of the original study, psychiatry clerkship students participated in two activities on the same day: one using a standardized patient and one using manikin-based simulation. Given that standardized patients are widely used in psychiatry education, we were hoping to demonstrate that manikin-based simulation was equally accepted by the students. Comparing standardized patients to manikin-based simulation, we found no significant differences in perceived effectiveness of the activities, ease in treating the manikin-based simulation or standardized patients as a real patient, enjoyment of the activities, or desire to participate in similar activities in the future [3].

We were hopeful that our successful activity could be replicated and utilized at other institutions. Additionally, we did not test knowledge acquisition and instead focused on student acceptance. Hence, the natural extension of this work was to expand to other institutions and test knowledge acquisition. We have subsequently partnered with other institutions and are presenting follow-up information here.

Sarah E. Baker

Sarah.Baker@UTSouthwestern.edu

University of Texas Southwestern Medical Center, Dallas, TX, USA

2 Dell Medical School at the University of Texas at Austin, Austin, TX, USA

3 Baylor Scott and White Health, Temple, TX, USA

4 Yale School of Medicine, New Haven, CT, USA
While Yale Medical School (Yale) continued to administer the activity to clerkship students, faculty at the University of Texas Southwestern Medical Center (UTSW) and Dell Medical School at the University of Texas at Austin (Dell) also adopted and administered the manikin-based simulation. In addition to a four-question opinion-based survey, students completed a twenty-question quiz prior to and after the learning activity. The study was reviewed by the institutional review boards at each site. It was deemed to be exempt at Yale and Dell and was expedited at UTSW.

UTSW had the largest group of participating students, with 116 students (94.3\%) participating in the pre-activity quiz and 117 students participating in the post-activity quiz (95.1\%). They had $83(67.5 \%)$ participating in the pre-activity survey and $105(85.4 \%)$ participating in the post-activity survey. Yale had 48 students participate in the pre-activity survey and quiz (79.2\%), and 25 students participate in the post-activity survey and quiz (52.1\%). Dell had a 100\% participation rate, with 29 students participating in both the pre-activity and post-activity surveys and quizzes. The average participation rates across all three schools for the pre- and post-activity quizzes were $91.2 \%$ and $82.4 \%$. Average participation rates for the preand post-activity survey were $82.2 \%$ and $79.2 \%$. Across all three sites, there was a significant increase in correct quiz responses related to the teaching activity $(p<0.001)$. Compared to the pre-activity expectations, the post-activity survey responses showed the manikin-based simulation activity was an effective teaching strategy $(p<0.001)$, enjoyable $(p<0.001)$, should be a part of the curriculum $(p=0.003)$, and that it was easy to treat the manikin as a real patient $(p<0.001)$.

Subjectively, students also commented that the manikinbased simulation activity was helpful when studying for standardized examinations and applicable to patient care. One student commented, "This was one of the best simulation cases I've ever been a part of. It was very effective in teaching the information covered, and it was extremely relevant to our exam." Another student commented, "I enjoyed the simulation case, and it definitely provided great learning points that I have already found applicable since this experience." Multiple students commented that they would like manikin-based 
simulation to be used more often in medical education. Overall, these results suggest that the manikin-based simulation activity was a useful instructional tool that aided in students' knowledge acquisition and was also enjoyable to students.

High-fidelity manikin-based simulation continues to be an underutilized teaching modality in psychiatry education [2]. The results of both studies illustrate that the manikin-based simulation activity developed at Yale is well received, improves knowledge, and can be successfully incorporated into clerkships at other institutions. We have recently developed an additional case involving a patient who presents to the emergency room in opioid withdrawal after naloxone administration.

In light of the COVID-19 pandemic, education with inperson patients has had limitations, especially in high-risk clinical areas like the emergency department (ED). Our manikin-based simulation activity involving an ED scenario offers an alternative educational method in a safe environment that students find enjoyable [4]. Additionally, manikin-based simulation is more cost-effective than standardized patients and avoids exposure and privacy concerns.
Declarations The study was reviewed by the institutional review boards at each site. It was deemed to be exempt at Yale and Dell Medical Schools and was expedited at the University of Texas Southwestern Medical Center.

Disclosures On behalf of the authors, the corresponding author states that there is no conflict of interest.

\section{References}

1. Bhalla IP, Wilkins KM, Moadel T, Wong AH, Trevisan LA, Fuehrlein B. Alcohol withdrawal and lithium toxicity: a novel psychiatric mannequin-based simulation case for medical students. MedEdPORTAL. 2017;13:10649.

2. Abdool PS, Nirula L, Bonato S, Rajji TK, Silver IL. Simulation in undergraduate psychiatry: exploring the depth of learner engagement. Acad Psychiatry. 2017;41(2):251-61. https://doi.org/10. 1007/s40596-016-0633-9.

3. Fuehrlein B, Bhalla I, Goldenberg M, Trevisan L, Wilkins K. Simulate to stimulate: manikin-based simulation in the psychiatry clerkship. Acad Psychiatry. 2020;44:82-5.

4. Tabatabai S. Simulations and virtual learning supporting clinical education during the COVID 19 pandemic. Adv Med Educ Pract. 2020;11:513-6. https://doi.org/10.2147/AMEP.S257750.

Publisher's Note Springer Nature remains neutral with regard to jurisdictional claims in published maps and institutional affiliations. 